\title{
Observatory Deployment and Characterization of SAPHIRA HgCdTe APD Arrays
}

\author{
Dani Atkinson ${ }^{a}$, Donald Hall ${ }^{a}$, Christoph Baranec ${ }^{a}$, Ian Baker ${ }^{b}$, Shane Jacobson ${ }^{a}$, and Reed \\ Riddle $^{c}$ \\ ${ }^{a}$ Institute for Astronomy, University of Hawai'i at Mānoa, Hilo, Hawai'i 96720-2700, USA; \\ ${ }^{b}$ Selex ES Ltd, Southampton, Hants, SO15 0LG, UK; \\ ${ }^{c}$ Division of Physics, Mathematics, and Astronomy, California Institute of Technology, \\ Pasadena, CA 91125, USA;
}

\begin{abstract}
We report the performance of Selex ES' SAPHIRA APD arrays from both laboratory characterization and telescope deployment. The arrays are produced using the MOVPE production method, allowing for solid state engineering and thus produce superior performance to similar liquid phase epitaxy efforts. With an avalanche gain slightly over 50 and read noise of $\sim 9 \mathrm{e}^{-}$, the detectors are easily capable of single-frame sub-electron read noise, and the 32 output readout and flexible windowing allow an excellent readout speed. Gain-corrected dark current/glow is found to be $10-20 \mathrm{e}^{-} / \mathrm{s}$ at low bias, and drops below basline at high avalanche gains. The detectors were also tested on-sky at both IRTF on Maunakea and the 1.5-m telescope at Palomar Observatory, demonstrating that the SAPHIRA is an ideal device for both tip-tilt NGS guiding and infrared lucky imaging, in the latter providing diffraction-limited resolution for the 3-meter IRTF without the benefit of adaptive optics correction.
\end{abstract}

Keywords: HgCdTe, mercury cadmium telluride, MCT, near infrared, NIR, MOVPE, avalanche photo diodes, APDs, tip-tilt, natural guide star, adaptive optics, lucky imaging

\section{INTRODUCTION}

The exceptionally low dark current, high quantum efficiency, and tunable cut-off wavelength of mercury cadmium telluride ( $\mathrm{HgCdTe})$ have brought it to the forefront of astronomical infrared detectors, most prolifically in the HAWAII series of large-format arrays used in a variety of astronomical instruments both ground- and spacebased. ${ }^{1}$ Early investigations into its breakdown properties found that it evidenced single-carrier avalanche behavior, i.e. that holes are virtually immobile relative to electrons. ${ }^{2}$ The resulting reduction in phonon scattering produces a gain-independent statistical excess noise, and was shown to circumvent existing theoretical limits on high gain avalanche multiplication. ${ }^{3}$ The deterministic avalanche and the absence of a breakdown effect in $\mathrm{HgCdTe}$ at high voltage make it promising a candidate for high-gain low-noise linear mode avalanche photo diodes (APDs) ${ }^{4,5}$ simultaneously allowing both individual photon counting and a large dynamic range. ${ }^{6}$ When the recorded low dark current of $\mathrm{HgCdTe}$ at cryogenic temperatures (e.g. Ref. ${ }^{7}$ ) are taken into account, $\mathrm{HgCdTe}$ APDs are theoretically capable of noiseless performance.

\section{SAPHIRA}

The current generation of SAPHIRA detectors are $320 \times 256 \times 24 \mu \mathrm{m}$ HgCdTe APD arrays with a 32-channel readout. The window register format of the readout integrated circuit (ROIC) allows for a variety of window sizes to take advantage of the full 32 channels. Although the chip is formatted to allow the use of the two $32 \mathrm{x}$ 256 wings at either end of the array to be used as reference pixels, all of the current SAPHIRA arrays are fully hybridized.

Further author information: (Send correspondence to D.A.)

D.A.: E-mail: atkinson@ifa.hawaii.edu, Telephone: (808) 932-2329

D.H.: E-mail: hall@ifa.hawaii.edu, Telephone: (808) 932-2360

High Energy, Optical, and Infrared Detectors for Astronomy VI, edited by Andrew D. Holland, James Beletic, Proc. of SPIE Vol. 9154, 915419 - (C) 2014 SPIE · CCC code: 0277-786X/14/\$18 - doi: 10.1117/12.2056807 


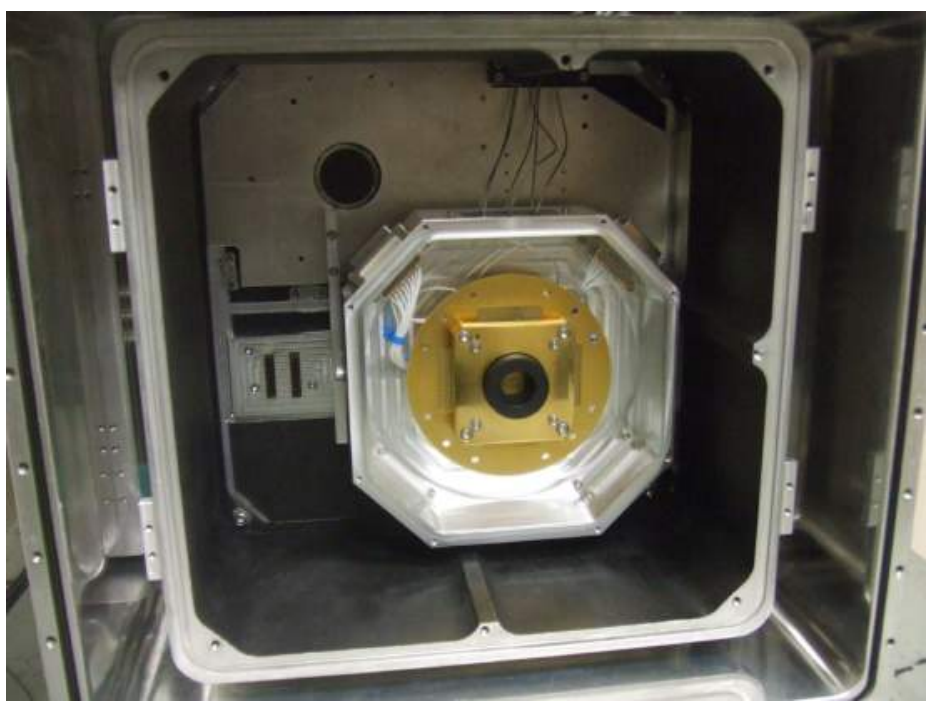

Figure 1. The inside of the KSPEC testbed, showing the detector mounting site. When in operation, an integrating sphere covers the detector's viewport, providing flat illumination from internal calibration LEDs.

The SAPHIRA arrays are manufactured using metal organic vapor phase epitaxy (MOVPE), which has only recently seen application to astronomical HgCdTe devices. MOVPE offers superior dark current and quantum efficiency to methods like liquid phase epitaxy (LPE), ${ }^{8}$ with a lower cost and greater reliability than either molecular beam epitaxy (MBE) or LPE. The MOVPE technique also has the advantage of successfully producing the n-on-p HgCdTe required for APD arrays.

All laboratory characterization of the SAPHIRA devices was performed in KSPEC (see Fig. 1). Originally a near-infrared cross-dispersed grating spectrometer, ${ }^{9} \mathrm{KSPEC}$ was repurposed for ultra-low noise detector characterization for the JWST Packaging and Manufacturing Technology program, where the background was shown to be at the level of a few photons/pixel/hour for $5 \mu \mathrm{m}$ cutoff material. ${ }^{10}$ KSPEC makes use of a closed-cycle cryocooler to reach temperatures as low as $20 \mathrm{~K}$, with PID-controlled internal electric heating providing fine-tuning and temperature stability. Infrared LEDs are mounted to an integrating sphere covering the detector's viewport, providing flat illumination of the detector surface for characterization uses, with masks are occasionally placed into the path for imaging tests.

The SAPHIRA devices are operated by a Generation III Leach controller, ${ }^{11}$ a modular system allowing configuration for a variety of astronomical detectors. Four ARC-46 eight channel IR video boards are used to receive all 32 outputs of the detector. A low-noise voltage supply board designed at Australian National University is used to provide operating and bias voltages, customized to produce negative voltages down to $-12 \mathrm{~V}$ for high bias operation. At present, the operating speed of the Leach controller is the bottleneck of the instrument, and the pixel rate is capped at $265 \mathrm{kHz}$.

Selex has provided to UH two devices, one a standard APD structure (2815), the other an experimental, proprietary bandgap structure (2775). While the two show similar performance in many respects including avalanche gain, the former shows a breakdown current at higher bias voltages that results in an increased dark current. Except where noted, this publication reports the results from the latter.

\subsection{MOVPE Development}

MOVPE of HgCdTe was originally developed for commercial thermal imaging by Selex ES, UK. It provides programmed control of the thicknesses, bandgaps, and doping levels on a $0.1 \mu \mathrm{m}$-scale to create a range of new device structures including APDs. Low-cost GaAs substrates provide a wafer-scale process suitable for large area arrays. There was initially concern that the low binding energies, ionic bond nature, and open lattice of $\mathrm{HgCdTe}$ would make MOVPE too prone to dislocation-related breakdown in APDs at high voltages, but the first devices 


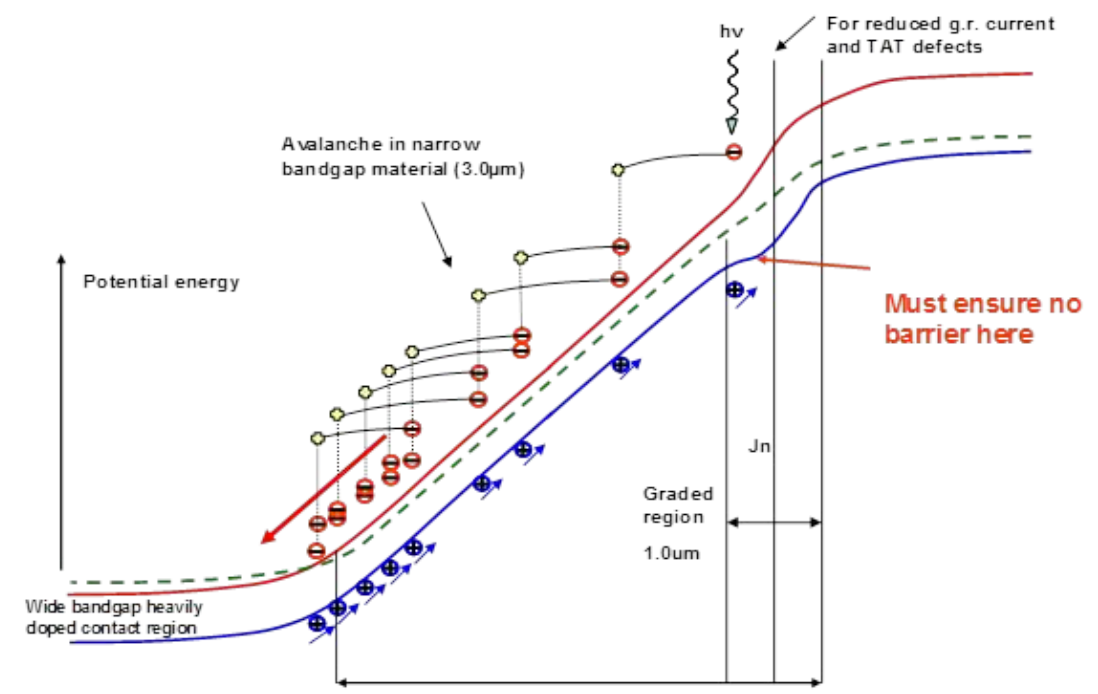

Figure 2. Band structure of MOVPE heterostructure APD array. The junction is placed in a wide band-gap buffer to reduce trap-assisted tunneling current and trap-related thermal current and to provide immunity from weak misfit dislocation loops (courtesy Selex ES).

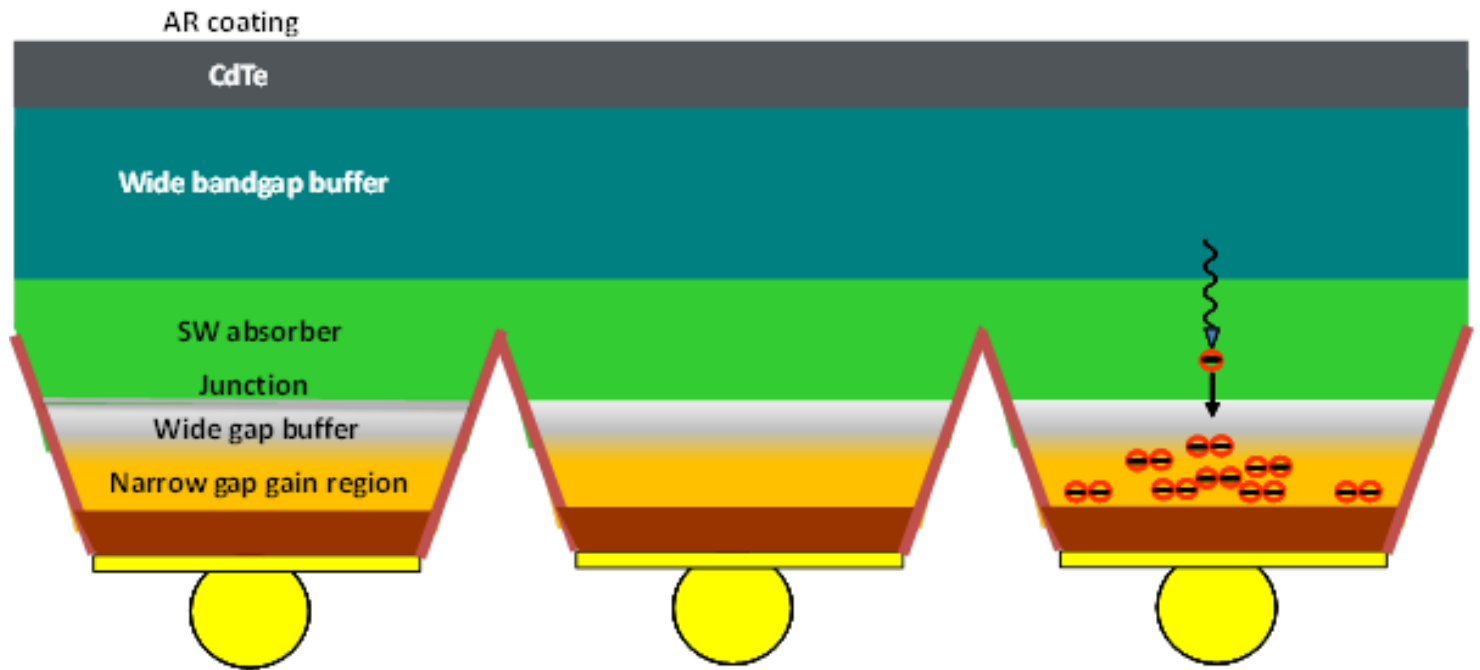

Figure 3. Schematic of MOVPE heterostructure APD array. The gain region uses a narrow bandgap for a higher gain relative to bias voltage. The absorber and the gain region are therefore decoupled so each can be optimized separately. The buffer layer between the two is a thick sequence of $\mathrm{HgTe}$ and CdTe cycles that turns over misfit dislocations arising from the crystal lattice mismatch of GaAs (courtesy Selex ES).

produced for ESO as part of the VLTI/GRAVITY effort showed that bandgap engineering (see Fig. 2) and a properly optimized device design (see Fig. 3) easily overcome the weak misfit dislocations, allowing detectors with a high optical absorption coefficient and efficient device structure. ${ }^{8}$

Each pixel is electrically isolated by a mesa slot that extends though the absorber to eliminate lateral collection (see Fig. 4). The optical design emphasizes trapping photons within the mesa cone to produce complete absorption. This ensures that stray photons do not scatter and contribute to the background current of other pixels, improving the optical crosstalk, modulated transfer function, and pixel inter-capacitance of the arrays. A combination of dry and wet etching is used to ensure the sidewall shape and quality. The sidewalls are then coated with a CdTe layer that is inter-diffused at high temperature. The widening of the bandgap around the 

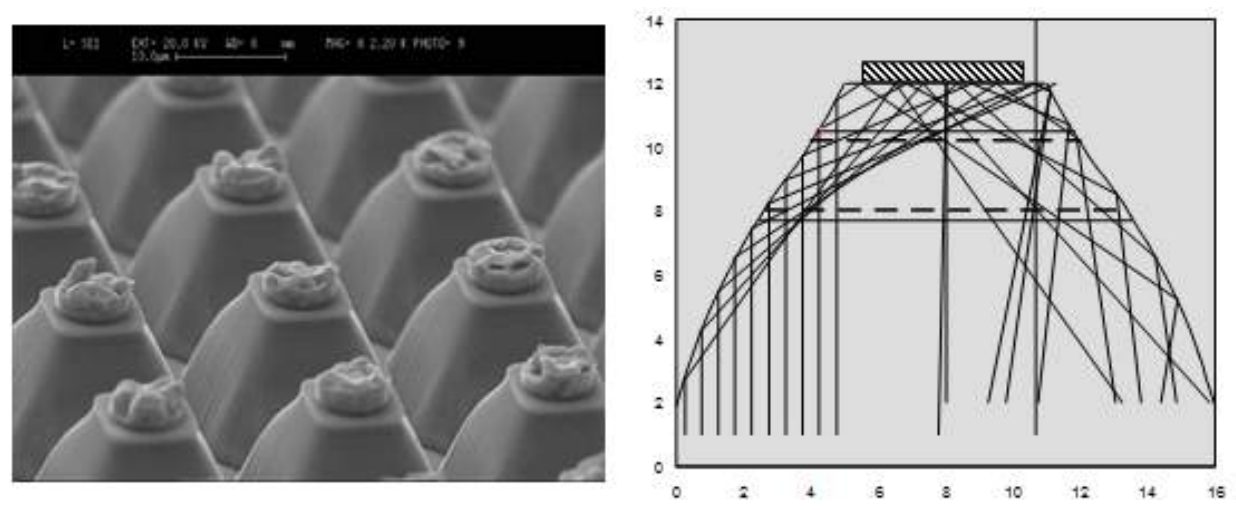

Figure 4. Photograph showing the mesa diode structure (left), and a ray tracing diagram demonstrating the trapping of incident photons (right). Rays represent photons entering the detector on parallel paths from the bottom of the diagram, and are internally reflected without scattering to adjacent pixels (courtesy Selex ES).

edges of the absorber effectively separates carriers from surface states and minimizes junction currents where the junction intercepts the sidewall. The high temperature anneal also promotes long term stability and immunity from life at high operational temperatures. $\mathrm{HgCdTe}$ is the only infrared material that permits so-called hetero-passivation and it has a strong impact on the reverse-bias breakdown in APDs.

The first generation devices essentially used Selex's thermal imaging technology with a design optimized for APDs. The arrays showed substantially better performance than similar LPE devices, particularly with respect to cosmetics at high voltages (see Sect. 2.4). The widening of the bandgap at the junction and the independent optimization of the absorber and gain region very effectively suppressed the number of defects caused by trapassisted tunneling. MOVPE has intrinsically lower dark current compared with $\mathrm{LPE}^{8}$ and the arrays work quite well at temperatures around $85 \mathrm{~K}$ compared with $40 \mathrm{~K}$ for LPE.

The MOVPE technology developed for thermal imaging has limitations for some science applications, particularly in low-temperature frequency response (see Sect. 2.3) and $J$-band QE, with the latter a result of buffer layer absorption (see Fig. 3 for a diagram of the buffer).

Development of the SAPHIRA APDs is ongoing, and funded by ESO and UKATC as well as UH. Future iterations of the device are targeting improvements in response time and $\mathrm{J}$ band performance. There is also interest in achieving higher breakdown voltages and higher overall QE by further optimization of the design and processing technology.

\subsection{Avalanche Gain \& Read Noise}

Using KSPEC's internal LED at $\lambda=1.7 \mu \mathrm{m}$, the avalanche gain of the SAPHIRA was measured for the complete range of bias voltages. The full $320 \times 256$ array was read out over a consistent time interval, dark subtracted, and the measurements normalized to a bias of $1.5 \mathrm{~V}$. The resulting gain curve is consistent with a linear $\mathrm{HgCdTe}$ avalanche, reaching a gain of 52 at a bias voltage of $13.5 \mathrm{~V}$ (see Fig. 5). At lower bias voltages the gain falls as a function of increasing pixel capacitance; this effect has been accounted for in the data.

Charge gain was also measured using the standard technique, fitting the Poisson statistical relationship between signal and variance to the measured per-pixel values of dark-subtracted ramps at unity gain. For the SAPHIRA APD in our setup, this gives a charge gain of $2.9 \mathrm{e}^{-} /$ADU and a read noise of $\sim 9 \mathrm{e}^{-}$. The read noise is lower than reported by the ESO investigation ${ }^{8}$ due to our lower pixel rate of $265 \mathrm{kHz}$ and correspondingly longer integration time. A linear gain of 9 is then sufficient to provide sub-electron read noise, at as little as $9.5 \mathrm{~V}$ bias.

\subsection{Frequency Response}

As stated in Sect. 2.1, when operated at lower temperatures the SAPHIRA detectors show a drop in frequency response on the order of milliseconds, evidenced as a decay curve towards the relevant signal. It was reported 


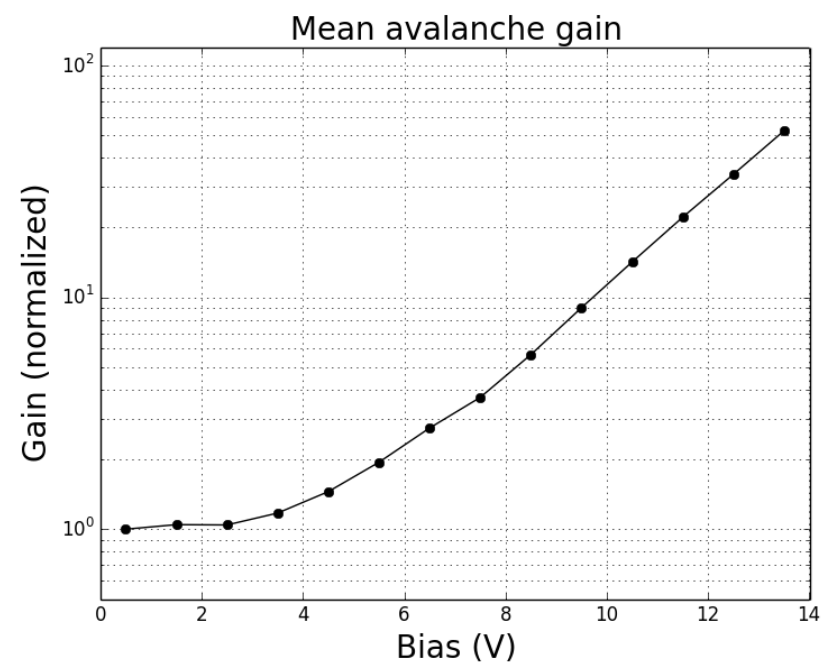

Figure 5. The mean avalanche gain across an engineering-grade SAPHIRA APD array, topping out at just over 50. The measurements have been corrected for rising capacitance at lower gain.

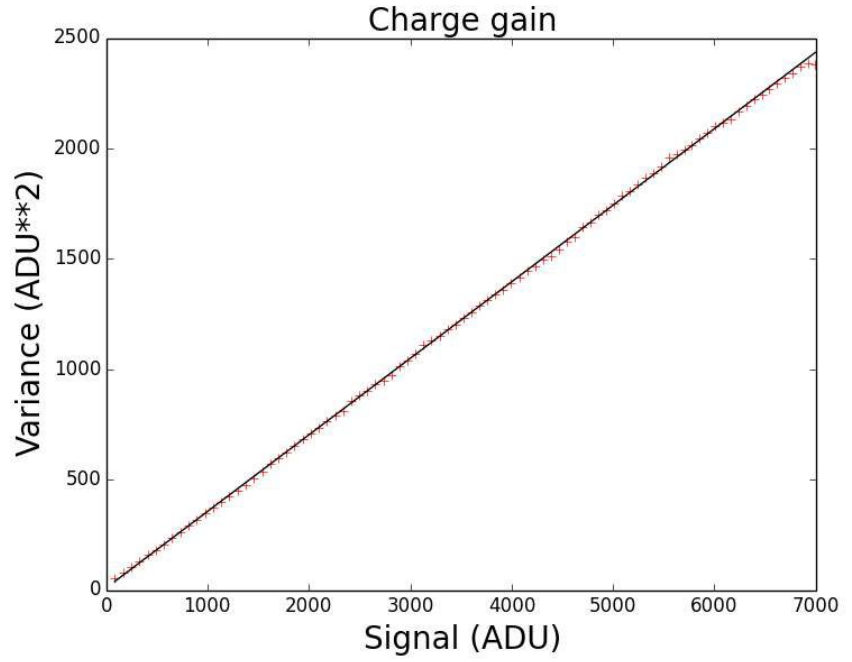

Figure 6. Signal vs. variance for the SAPHIRA APD array (red crosses), with the fitted line (black) yielding a charge gain of $2.9 \mathrm{e}^{-} / \mathrm{ADU}$ and a read noise of $9 \mathrm{e}^{-} \mathrm{rms}$. 


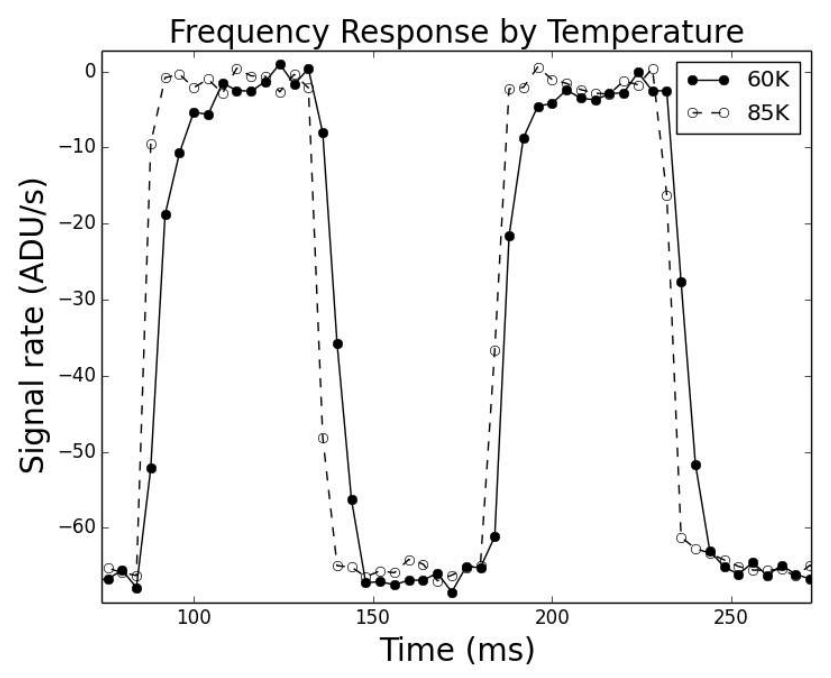

Figure 7. The frequency response of the SAPHIRA detector compared for $60 \mathrm{~K}$ and $85 \mathrm{~K}$, showing the drop in response time at lower temperature. The individual points are at time increments of $2 \mathrm{~ms}$.

recently that at $60 \mathrm{~K}$ the response may be as slow as $30 \mathrm{~ms}$, which shrinks below detection at $85 \mathrm{~K} .^{8}$ We conducted the same measurement by operating the detector at unity gain with the calibration LED switched at $200 \mathrm{~Hz}$ with a confirmed ns-scale rise time, CDS subtracting the frames and averaging together adjacent pairs of frames to reduce noise, and found a response of $\sim 7 \mathrm{~ms}$ at $60 \mathrm{~K}$ and $<1 \mathrm{~ms}$ at $85 \mathrm{~K}$, partially confirming the earlier results (see Fig. 7). Device 2815 showed similar behavior. Selex posits that this effect is due to the trapping of electrons in the absorber, and future iterations will attempt to reduce this trap density.

\subsection{Dark Current \& Glow}

Dark current was measured in KSPEC with $1 \mathrm{~Hz}$ full-frame data, trimming recorded ramps well short of where non-linear behavior appears, measuring the mean difference in counts and dividing by the time elapsed. Although electronic glow from the ROIC was initially the dominant photon source, it was reduced by a factor of $\sim 25$ dropping the operating voltages provided to the detector (and which does not otherwise alter its performance). The measured dark currents in this operating condition are shown in Tab. 1. At low bias voltages, the mean dark current is $\sim 15 \mathrm{e}^{-} s$, and grows to $\sim 250 \mathrm{e}^{-} s$ at higher bias. As reported in Sect. 2.2, the avalanche gain increases by a factor of 60 over this interval, but the dark current sees only a partial amplification due to 'dark' electrons originating at varying depths in the gain region. The combined effect is that once the dark current is adjusted for avalanche gain, it is functionally lower at higher bias voltages relative to absorbed photoelectrons.

\begin{tabular}{|l|l|l|l|}
\hline Bias & Raw & $* 2.9 \mathrm{e}^{-} / \mathrm{ADU}$ & Gain-corrected \\
\hline $0.5 \mathrm{~V}$ & $3.27 \pm 0.19 \mathrm{ADU} / \mathrm{s}$ & $9.4 \pm 0.6 \mathrm{e}^{-} / \mathrm{s}$ & $9.4 \pm 0.6 \mathrm{e}^{-} / \mathrm{s}$ \\
\hline $3.5 \mathrm{~V}$ & $6.28 \pm 1.11 \mathrm{ADU} / \mathrm{s}$ & $18.1 \pm 3.2 \mathrm{e}^{-} / \mathrm{s}$ & $15.5 \pm 2.7 \mathrm{e}^{-} / \mathrm{s}$ \\
\hline $7.5 \mathrm{~V}$ & $23.2 \pm 5.3 \mathrm{ADU} / \mathrm{s}$ & $66.8 \pm 15.4 \mathrm{e}^{-} / \mathrm{s}$ & $18.1 \pm 4.2 \mathrm{e}^{-} / \mathrm{s}$ \\
\hline $11.5 \mathrm{~V}$ & $91.7 \pm 21.7 \mathrm{ADU} / \mathrm{s}$ & $264 \pm 62 \mathrm{e}^{-} / \mathrm{s}$ & $5.0 \pm 1.2 \mathrm{e}^{-} / \mathrm{s}$ \\
\hline
\end{tabular}

Table 1. Dark current measurements for a representative sample of SAPHIRA array 2775. Population standard deviations are shown for the pixels sampled. The last column is adjusted for the respective avalanche gain of that bias voltage. The dark current doesn't experience the full benefit of the avalanche gain, and is effectively lower relative to the photon signal at the high end of the range.

The map of per-pixel dark current behavior shows the array to be fairly uniform, although some residual glow is apparent at the bottom (see Fig. 8). The vast majority of pixels are well-behaved (hence the mean dark 


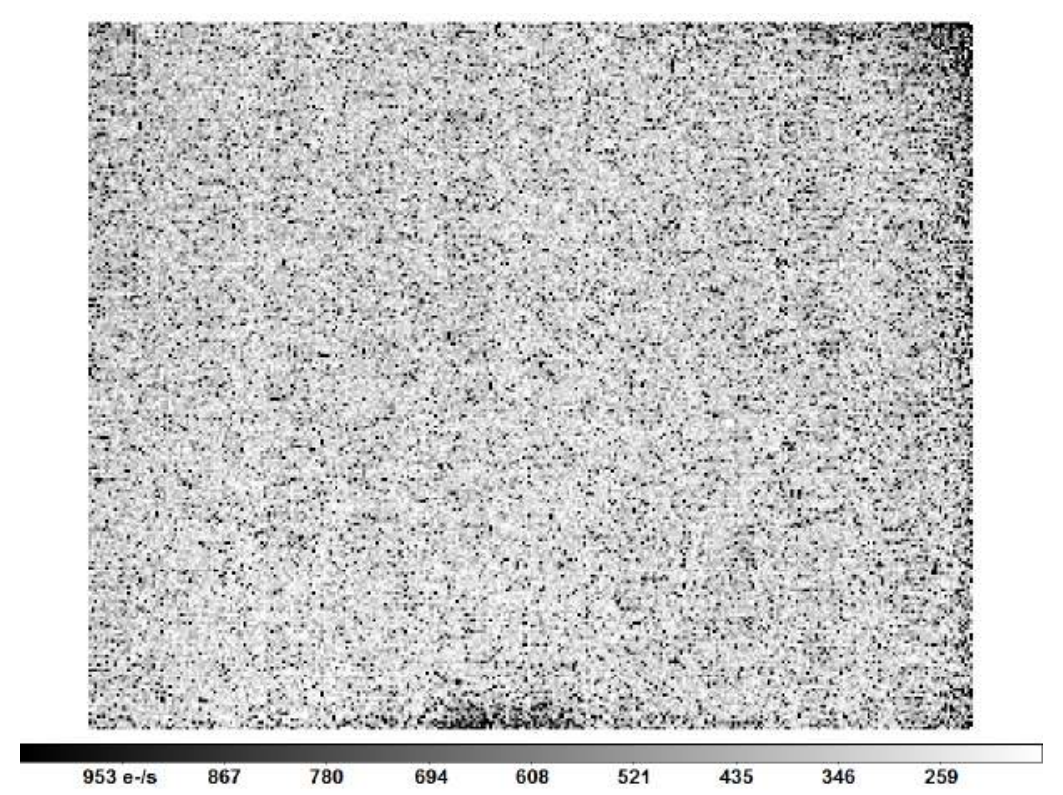

Figure 8. Map of the dark current across the array at a bias of $-11.5 \mathrm{~V}$.

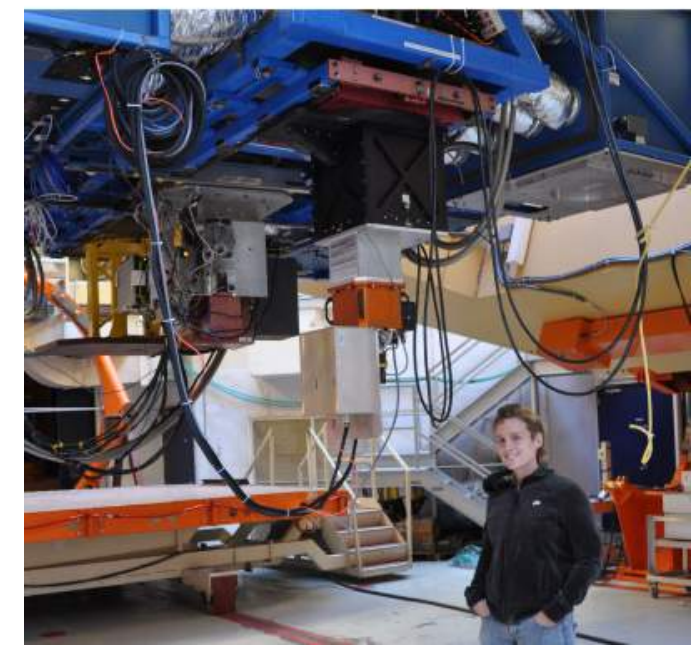

Figure 9. The GLS cryostat containing the SAPHIRA detector (orange box at center) mounted on IRTF, with the author as a scale reference.

current being close to the low end of the scale, and the standard deviation of the population relatively low), while the 'hot' pixels are scattered randomly.

\section{IRTF TESTING}

The first on-sky observatory test was conducted at the 3-meter NASA Infrared Telescope Facility at Maunakea Observatory April 30 and May 1 2014, with the SAPHIRA detector in a GLS Sterling Cooler Cryostat occupying the telescope's Cassegrain focus (see Fig. 9). Images were taken in the $H$-band. The performance of the detector was evaluated in two operating modes: as a centroid tracker for low-order wavefront sensing; and as a lucky imager, taking advantage of its rapid readout speed. 


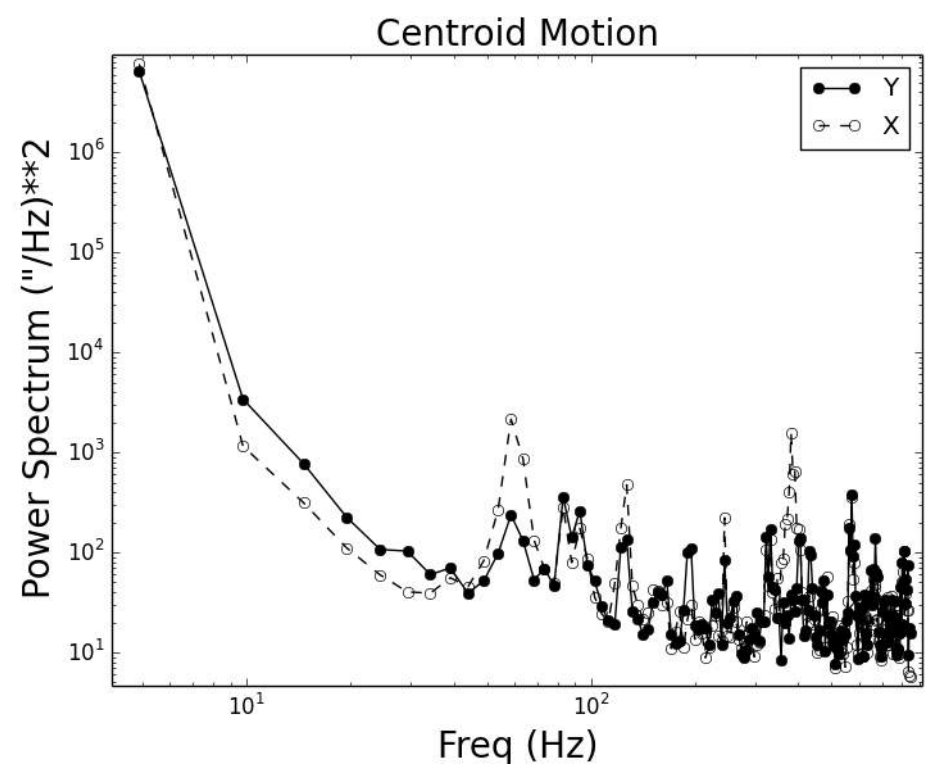

Figure 10. An unsmoothed power spectrum of centroid motion from IRTF observations of 24 LMi. The $f^{-\frac{5}{3}}$ noise slope down to $\sim 200 \mathrm{~Hz}$ is from atmospheric seeing, while the peak at $60 \mathrm{~Hz}$ is a mechanical vibration.

\subsection{Centroid Tracking for Tip-Tilt}

For the purpose of NGS centroid tracking, the detector was configured to read out a $64 \mathrm{x} 64$ pixel window at $1.7 \mathrm{kHz}$, covering a 2.8 " $\times 2.8$ " field centered on the singleton star $24 \mathrm{LMi}(H=4.946) .{ }^{12}$ No correction servo was implemented; this is a simple proof of concept for the detector in future uses as a tip-tilt sensor. A power spectrum of the centroid is shown in Fig. 10, with the dominant signals being the $f^{-\frac{5}{3}}$ atmospheric seeing continuum and a mechanical vibration at $60 \mathrm{~Hz}$. (The source of the mechanical vibration was isolated and the effect mitigated for future observations.)

\subsection{Lucky Imaging}

Given the readout speed of the detector, lucky imaging is an obvious application. The seeing on Mauna Kea was very good for the two nights observed as reported by the adjacent Canada-France-Hawai'i Telescope, hovering around 0.4" for much of the first night with intermittent declines into the arcsecond range, and was reported at near 0.3 " during the second. Fig. 11 shows the results as applied to the $1.7 \mathrm{kHz} 24 \mathrm{LMi}$ data. Both images were assembled by $2 \times 2$ block replication of all pixels, measurement of the centroid of the object to determine offset, and concentric composition of the relevant CDS frames. In the full integration, the aberrations of IRTF are partially visible in the lobes around the star's center, and the FWHM of the composite object is $\sim 0.3$ ", consistent with the night's seeing reports. The lucky image shows the composite of the top 10 percent of images, selected by a basic peak intensity test using the central pixels, and presents a diffraction-limited PSF with partial rings. The FWHM of the PSF core is 0.12 ", the diffraction limit for the 3-meter telescope in the H-band. The SAPHIRA is thus capable of near-infrared diffraction-limited lucky imaging.

As a more complex test, the full 14.2" x 11.4" field was used to image a section of M3, read out at $100 \mathrm{~Hz}$ (see Fig. 12). In this case, the image is partially blurred horizontally as the slower readout is less able to freeze out the $60 \mathrm{~Hz}$ field motion. Still, the FWHM improves from $\sim 0.4$ " in the seeing-limited image to $\sim 0.2$ " in the lucky image.

\section{PALOMAR SIXTY-INCH DEPLOYMENT}

After IRTF, the detector was also given a test deployment at the Palomar Sixty-Inch (P60) telescope from June 13-19, a 1.5-meter visible/NIR telescope housing the Robo-AO automated LGS AO imager. ${ }^{13,14}$ This installation 

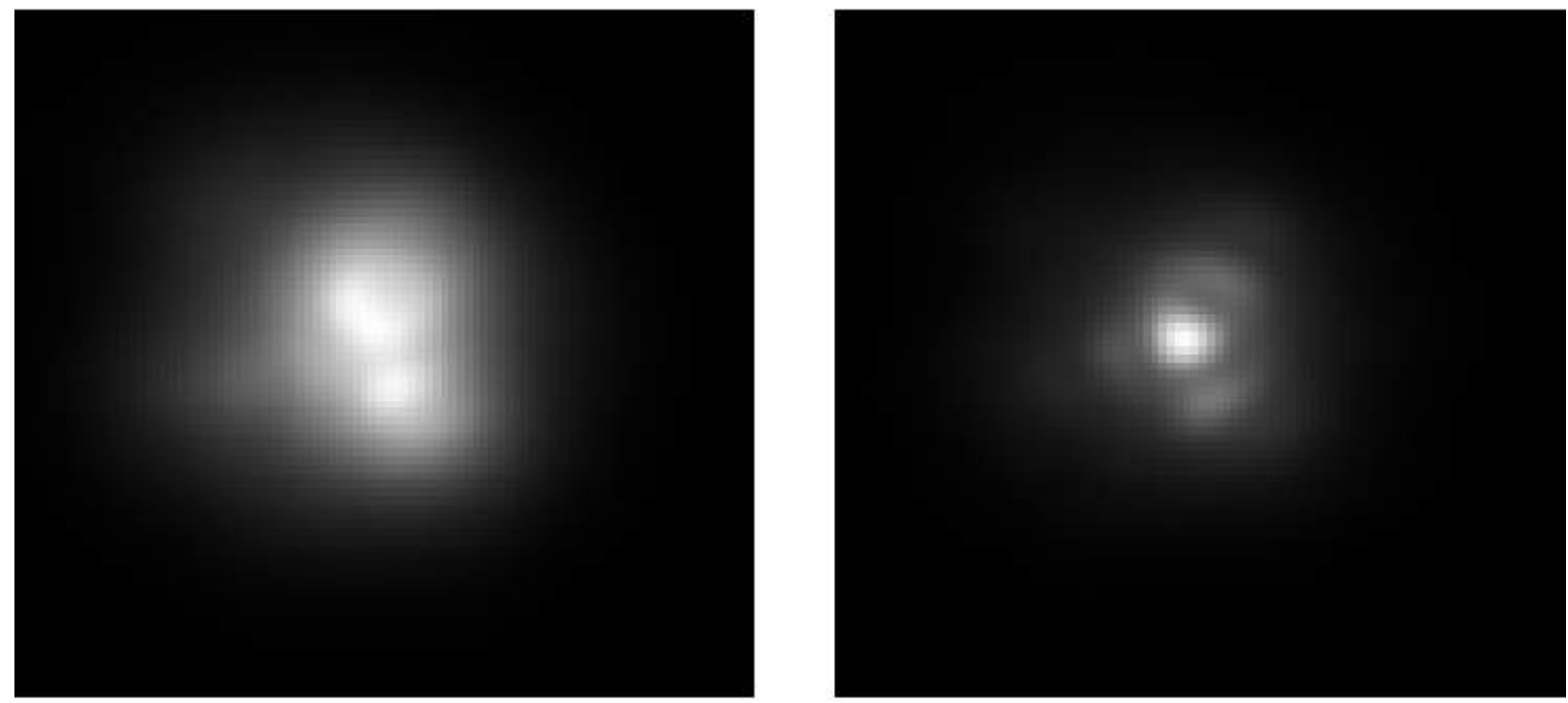

Figure 11. Lucky imaging results from 2.8" x 2.8" IRTF observations of 24 LMi. The full integration (left) contains all images in the series, with only shift-and-add correction applied. The lobes visible in the PSF are due to aberrations in IRTF optics. The lucky image (right) contains the best 10 percent of images selected by core intensity, and is diffractionlimited with a partial diffraction ring visible.
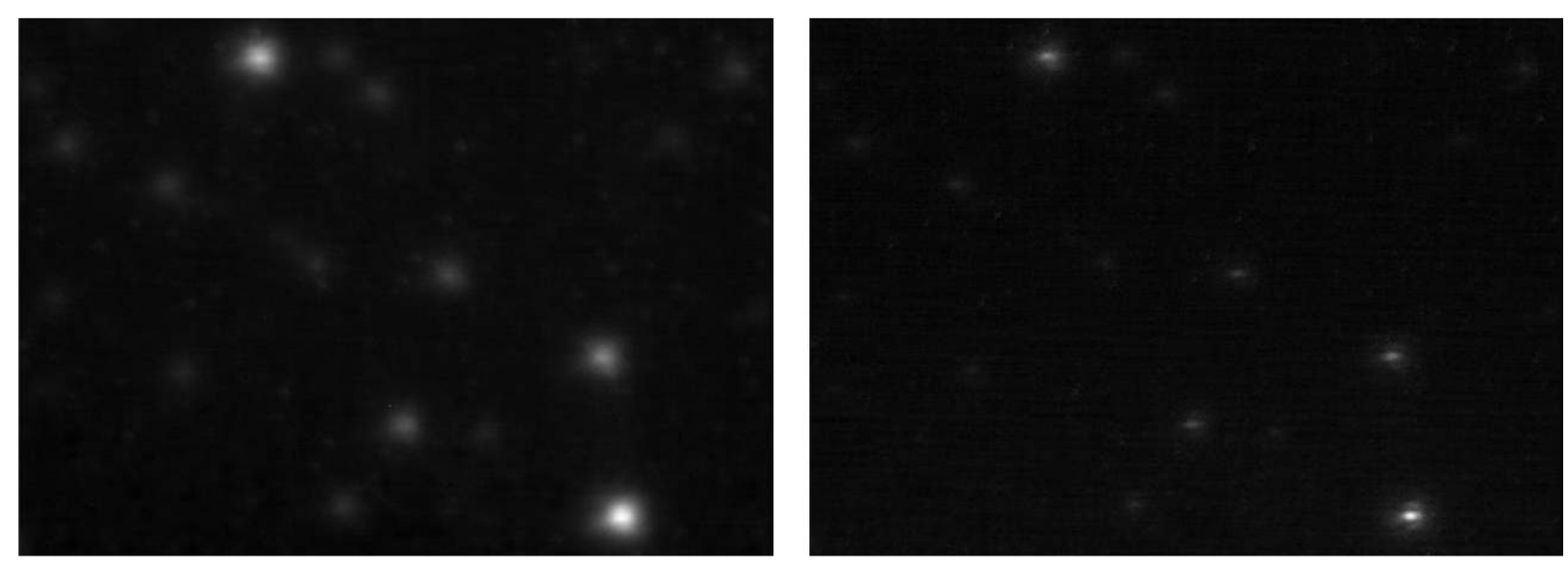

Figure 12. Lucky imaging results from 14.2" x 11.4" IRTF observations of a section of M3 at 100Hz. Similar to Fig. 11, the full integration (left) has only vertical/horizontal correction, while the lucky image (right) contains the best $0.2 \%$ of images. As the partial image smearing demonstrates, the $100 \mathrm{~Hz}$ readout is slightly less capable at freezing seeing conditions, although the result is still close to diffraction-limited. 


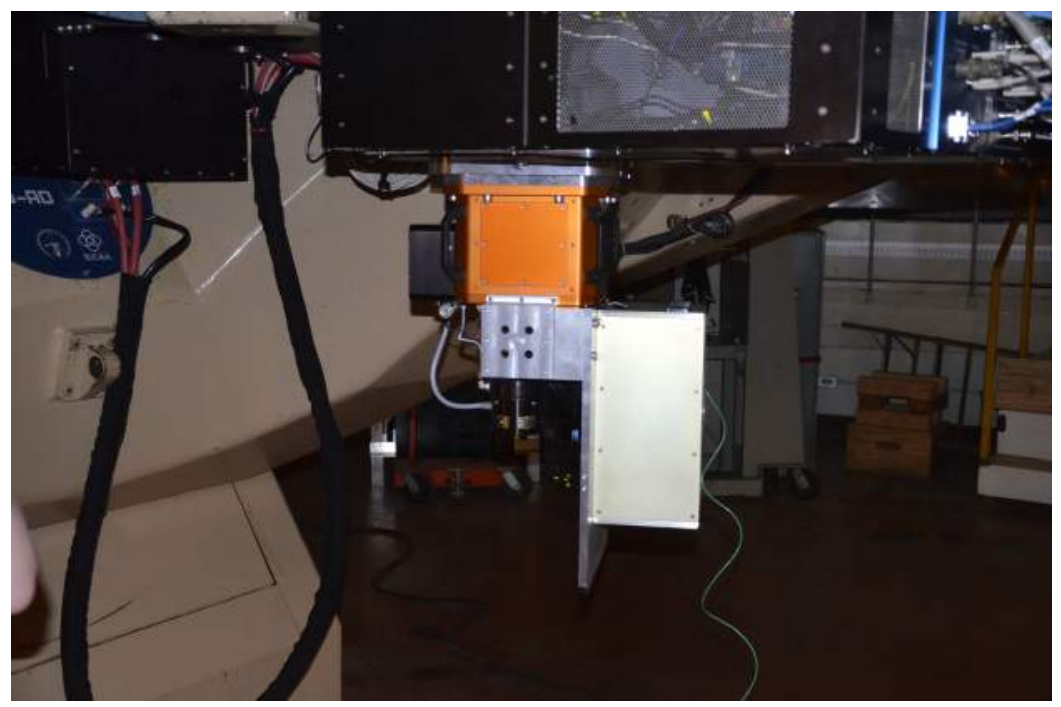

Figure 13. The GLS cryostat with SAPHIRA detector (orange box at center) mounted on the underside of the Robo-AO instrument at P60.
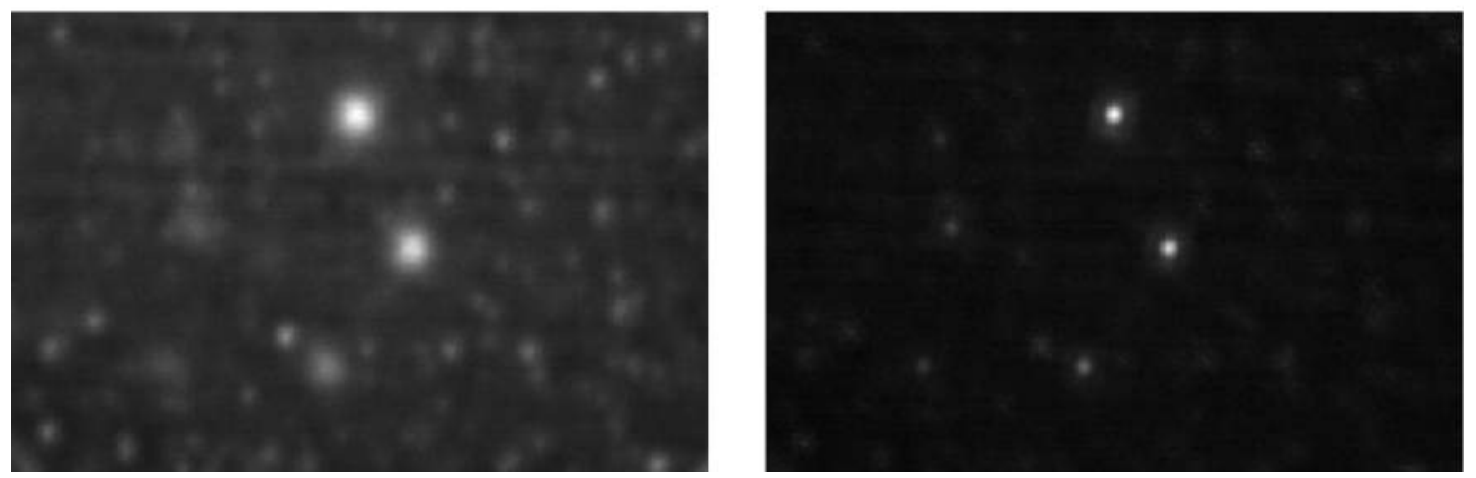

Figure 14. M3 lucky imaging results from the initial P60 deployment. Shown is a subsection of the full field, approximately 13.7" by 9.3". The full integration (left) received only shift-and-add correction, while the lucky image (right) is a $10 \%$ selection showing a diffraction-limited profile with visible Airy disks.

is the first step in future integration with Robo-AO.

\subsection{Test Deployment \& Selected Images}

The SAPHIRA was mounted to the NIR port of Robo-AO, operated at $85 \mathrm{~K}$ and used to take images alongside the system's existing EMCCD camera. Astronomical images were taken on the night of June 18, during which the P60 seeing monitor reported a mean seeing of $\sim 2$ ", though the Robo-AO wavefront correction was active. A lucky imaging test similar to that performed at IRTF was conducted, again on M3. Initial results are shown in Fig. 14, and bear a resemblance to the IRTF results, but more detailed reduction and analysis is needed.

\subsection{Future Commissioning on Robo-AO}

The SAPHIRA array is slated for further use on Robo-AO/P60 through fall of this year. During this summer the APD array will be integrated into the Robo-AO system to provide NIR tip-tilt NGS guidance, supplementing the existing LGS adaptive optics correction (as LGS is incapable of providing tip-tilt guidance) and acting as a proof-of-concept for similar operation on larger telescopes. Robo-AO includes an EMCCD for visible lucky 
imaging applications, and the addition of a SAPHIRA APD will extend this capability to the near infrared. Scientifically, this system will improve the performance of Robo-AO in its high-resolution high-cadence observations, particularly in the ongoing companion survey of Kepler Object of Interest host stars. ${ }^{15}$

\section{CONCLUSIONS}

The SAPHIRA arrays' dark current and cosmetic quality clearly establishes MOVPE as the production method of choice for n-on-p HgCdTe. As APDs, their exceptional gain performance makes them easily capable of subelectron read noise, and even with a controller-limited $265 \mathrm{kHz}$ pixel rate they can provide not only accurate tip-tilt measurement but also diffraction-limited lucky imaging in the near infrared. Future SAPHIRA development will focus on improving the low-temperature frequency response, $J$-band QE, and dark current. In addition to tiptilt and lucky imaging, SAPHIRA is also an ideal detector for NIR high time-resolution observations, and the MOVPE technology should allow relatively rapid advancement to megapixel-format arrays that will function as science detectors.

\section{ACKNOWLEDGMENTS}

The author is supported in her studies by a NASA Space Technology Research Fellowship, grant \#NNX 13AL75H. Development of the SAPHIRA detectors and their characterization at UH is sponsored by NSF ATI award AST 1106391 and NASA ROSES APRA award \#NNX 13AC13G.

\section{REFERENCES}

1. R. Blank, S. Anglin, J. W. Beletic, Y. Baia, S. Buck, S. Bhargava, J. Chen, D. Cooper, M. Eads, M. Farris, D. N. B. Hall, K. W. Hodapp, W. Lavelle, M. Loose, G. Luppino, E. Piquette, R. Ricardo, T. Sprafke, B. Starr, M. Xu, and M. Zandian, "The HxRG Family of High Performance Image Sensors for Astronomy," in Solar Polarization 6, J. R. Kuhn, D. M. Harrington, H. Lin, S. V. Berdyugina, J. Trujillo-Bueno, S. L. Keil, and T. Rimmele, eds., Astronomical Society of the Pacific Conference Series 437, p. 383, Apr. 2011.

2. C. T. Elliot, N. T. Gordon, R. S. Hall, and G. Crimes, "Reverse breakdown in long wavelength lateral collection $C d_{x} H g_{1-x}$ Te diodes," Journal of Vacuum Science Er Technology A 8, pp. 1251-1253, Mar./Apr. 1990.

3. J. D. Beck, C.-F. Wan, M. A. Kinch, and J. E. Robinson, "MWIR HgCdTe avalanche photodiodes," in Society of Photo-Optical Instrumentation Engineers (SPIE) Conference Series, R. E. Longshore, ed., Society of Photo-Optical Instrumentation Engineers (SPIE) Conference Series 4454, pp. 188-197, Nov. 2001.

4. J. D. Beck, C.-F. Wan, M. A. Kinch, J. E. Robinson, P. Mitra, R. E. Scritchfield, F. Ma, and J. C. Campbell, "The HgCdTe electron avalanche photodiode," in Infrared Detector Materials and Devices, R. E. Longshore and S. Sivananthan, eds., Society of Photo-Optical Instrumentation Engineers (SPIE) Conference Series 5564, pp. 44-53, Oct. 2004.

5. M. A. Kinch, "A Theoretical Model for the HgCdTe Avalanche Photodiode," Journal of Electronic Materials 37, p. 1453, 2008.

6. J. D. Beck, R. Scritchfield, P. Mitra, W. Sullivan, A. D. Gleckler, R. Strittmatter, and R. Martin, "Linear Mode Photon Counting with the Noiseless Gain HgCdTe e-APD," in Advanced Photon Counting Techniques $V$, M. A. Itzler and J. C. Campbell, eds., Society of Photo-Optical Instrumentation Engineers (SPIE) Conference Series 8033, Nov. 2011.

7. K. R. Kulas, I. S. McLean, and C. C. Steidel, "Performance of the HgCdTe detector for MOSFIRE, an imager and multi-object spectrometer for Keck Observatory," in Society of Photo-Optical Instrumentation Engineers (SPIE) Conference Series, Society of Photo-Optical Instrumentation Engineers (SPIE) Conference Series 8453, July 2012.

8. G. Finger, I. Baker, D. Alvarez, D. Ives, L. Mehrgan, M. Meyer, and J. Stegmeier, "NIR HgCdTe Avalanche Photodiode Arrays for Wavefront Sensing and Fringe Tracking," in Optical and IR Telescope Instrumentation and Detectors, Scientific Detector Workshop, Oct. 2013.

9. K.-W. Hodapp, J. L. Hora, E. Irwin, and T. Young, "KSPEC-a near-infrared cross-dispersed spectrograph," Publications of the Astronomical Society of the Pacific 106, pp. 87-93, Jan. 1994. 
10. D. N. Hall, K.-W. Hodapp, D. L. Goldsmith, C. A. Cabelli, A. K. Haas, L. J. Kozlowski, and K. Vural, "Characterization of lambdac $=5 \mathrm{um} \mathrm{Hg:Cd:Te} \mathrm{arrays} \mathrm{for} \mathrm{low-background} \mathrm{astronomy,"} \mathrm{in} \mathrm{Optical} \mathrm{and}$ IR Telescope Instrumentation and Detectors, M. Iye and A. F. Moorwood, eds., Society of Photo-Optical Instrumentation Engineers (SPIE) Conference Series 4008, pp. 1268-1279, Aug. 2000.

11. R. W. Leach and F. J. Low, "CCD and IR array controllers," in Optical and IR Telescope Instrumentation and Detectors, M. Iye and A. F. Moorwood, eds., Society of Photo-Optical Instrumentation Engineers (SPIE) Conference Series 4008, pp. 337-343, Aug. 2000.

12. R. M. Cutri, M. F. Skrutskie, S. van Dyk, C. A. Beichman, J. M. Carpenter, T. Chester, L. Cambresy, T. Evans, J. Fowler, J. Gizis, E. Howard, J. Huchra, T. Jarrett, E. L. Kopan, J. D. Kirkpatrick, R. M. Light, K. A. Marsh, H. McCallon, S. Schneider, R. Stiening, M. Sykes, M. Weinberg, W. A. Wheaton, S. Wheelock, and N. Zacarias, 2MASS All Sky Catalog of point sources., June 2003.

13. C. Baranec, R. Riddle, N. M. Law, A. N. Ramaprakash, S. P. Tendulkar, K. Bui, M. P. Burse, P. Chordia, H. K. Das, J. T. C. Davis, R. G. Dekany, M. M. Kasliwal, S. R. Kulkarni, T. D. Morton, E. O. Ofek, and S. Punnadi, "Bringing the Visible Universe into Focus with Robo-AO," Journal of Visual Experiments 72, p. 50021, Feb. 2013.

14. C. Baranec, R. Riddle, N. M. Law, A. N. Ramaprakash, S. Tendulkar, K. Hogstrom, K. Bui, M. Burse, P. Chordia, H. Das, R. Dekany, S. Kulkarni, S. Punnadi, and R. Smith, "High-efficiency autonomous laser adaptive optics." in press, 2014.

15. N. M. Law, T. Morton, C. Baranec, R. Riddle, G. Ravichandran, C. Ziegler, J. A. Johnson, S. P. Tendulkar, K. Bui, M. P. Burse, H. K. Das, R. G. Dekany, S. Kulkarni, S. Punnadi, and A. N. Ramaprakash, "Robotic Laser-Adaptive-Optics Imaging of 715 Kepler Exoplanet Candidates using Robo-AO," ArXiv e-prints, Dec. 2013. 